\title{
Tot geboren. Babys in Film, Literatur und Internet
}

In ihrer Autobiografie Inside out (2019) machte die US-amerikanische Schauspielerin Demi Moore öffentlich, dass sie 2004 im sechsten Schwangerschaftsmonat ein Kind verloren hatte. Kurz zuvor hatte Michelle Obama in ihrem Weltbestseller Becoming (2018) über eine Fehlgeburt Ende der 199oer Jahre berichtet, und Meghan Markle publizierte am 25. November 2020 in der New York Times einen Bericht über eine Fehlgeburt, die sie im Sommer 2020 erlitt. Diese Veröffentlichungen manifestieren auf exemplarische Weise, wie autobiografische Erzählungen heute zur Enttabuisierung von fehlgeschlagenen Schwangerschaften beitragen.

Gemeinhin unterscheidet man zwischen Fehlgeburt und Totgeburt. Die entsprechenden rechtlichen Kriterien sind von Land zu Land verschieden, sie betreffen die Anzahl der Schwangerschaftswochen und/oder das Gewicht des toten Kindes. In Österreich beispielsweise wird ein tot geborenes oder während der Geburt verstorbenes Kind als Totgeburt klassifiziert, wenn es mindestens 500 Gramm schwer ist; wiegt es weniger, so handelt es sich um eine Fehlgeburt. In Deutschland und in der Schweiz wird alternativ hierzu auch die Anzahl der Schwangerschaftswochen berücksichtigt: Von Totgeburt wird gesprochen, wenn ein Kind entweder mindestens 500 Gramm schwer ist oder wenn die 22. bzw. 24. Schwangerschaftswoche (Schweiz bzw. Deutschland) erreicht ist. Handelt es sich gemäß diesen Definitionen um eine Totgeburt, so besteht Meldepflicht, das heißt das Kind wird behördlich erfasst und ins Geburtenregister eingetragen, womit auch die Elternschaft vermerkt ist. In Deutschland, Österreich und der Schweiz kann eine solche Eintragung auch bei Fehlgeburten erfolgen.

In Großbritannien wurde die Trennlinie zwischen Fehlgeburt und Totgeburt in den 199oer Jahren von der 28. auf die 24. Schwangerschaftswoche herabgesetzt, was einen statistischen Anstieg von Totgeburten zur Folge hatte. ${ }^{1}$ Andere Länder unterscheiden nicht strikt zwischen Fehlgeburt und Totgeburt. In den USA werden tote Kinderbehördlich erfasst, wenn sie entwederüber $35^{\circ}$ Gramm schwer sind oder wenn sie die 20. Schwangerschaftswoche erreicht haben; in Australien liegt die entsprechende Gewichtsgrenze bei 400 Gramm. - All

1 Die Zahl der tot geborenen Kinder stieg dabei von 4,3 auf 5,7 pro eintausend Geburten an. Vgl. Samantha Murphy: Lost Futures. Stillbirth and the Social Construction of Grief. Saarbrücken 2010, S. 9 . 
diese Zahlen machen deutlich, dass die Definition von Totgeburt eine soziale Konstruktion und damit eine veränderbare Größe ist. Insbesondere anhand der Variable der Schwangerschaftswochen artikuliert sich die ganze Vehemenz des biopolitischen Diskurses, welcher den Beginn des Lebens und den Status des Todes zu definieren und letztlich zu kontrollieren sucht.

Statistiken der WHO machen deutlich, dass die Anzahl tot geborener Kinder in einkommensschwachen Ländern wie z. B. in der Republik Tschad, der Elfenbeinküste oder der Zentralafrikanischen Republik sehr viel höher ist als in Industriestaaten. ${ }^{2}$ Faktoren wie medizinische Versorgung, Ernährungslage und Geschlechterpolitik spielen dabei eine wichtige Rolle. In den westlichen Industriestaaten liegt die Anzahl tot geborener Kinder, unter Berücksichtigung numerischer Erfassungsdifferenzen und entsprechender statistischer Schwankungen, bei ca. 4 bis 6 pro eintausend Geburten. Als Gründe für den Tod eines Kindes im Mutterleib macht die Medizin in unseren Breitengraden u. a. Plazenta-Insuffizienz, Stoffwechselstörungen, Infektionen oder Nabelschnurumschlingungen geltend. Nichtsdestotrotz gibt es für etwa 30 bis $50 \%$ der Fälle keine medizinische Erklärung.

Das deutsche Wort „Totgeburt" macht, im Gegensatz etwa zum weicheren englischen „stillbirth/stillborn“, einen harten, unpersönlichen Eindruck. Es bezeichnet den Moment, in dem Anfang und Ende des Lebens am unerbittlichsten zusammenfallen. Verliert man ein Kind im fortgeschrittenen Schwangerschaftsstadium, so gilt es Abschied zu nehmen: Abschied von dem Wunsch nach diesem Kind, Abschied von einem vielleicht schon eingerichteten Kinderzimmer und anderen bereits besorgten Utensilien, Abschied von den Vorstellungen, die man sich gemacht hat - und Abschied von dem Kind selbst, Abschied von dessen kleinem toten Körper.

Gesellschaftlich war das Thema lange tabuisiert. So finden sich beispielsweise in der Literatur zur Geburtsvorbereitung nach wie vor kaum Hinweise darauf, dass ca. $0.4 \%$ aller Schwangerschaften mit einem tot geborenen Kind enden. Vielen werdenden Eltern ist diese Möglichkeit daher kaum bewusst, und in den angesprochenen autobiografischen Texten von Müttern tot geborener Kinder wird denn auch immer wieder die Frage gestellt: Wieso hat uns niemand gesagt, dass so etwas passieren kann? - Eine besondere Herausforderung stellt sich für schwangere Frauen, wenn das Kind im Mutterleib

2 Vgl online: https://www.who.int/reproductivehealth/topics/maternal_perinatal/stillbirth/en/ sowie https://www.who.int/reproductivehealth/topics/maternal_perinatal/stillbirth/Press_ release_stillbirths_2011/en/ sowie https://www.who.int/publications/i/item/9789241511223 (letzter Zugriff jeweils: 23.11.2021). 
stirbt und sie kurzfristig vor die Wahl gestellt werden, das tote Kind qua Kaiserschnitt oder auf natürlichem Weg zur Welt zu bringen.

Parallel zur Aufmerksamkeit, die das Thema heute in autobiografischen Texten findet, entstehen seit einigen Jahren vermehrt Einrichtungen, die betroffene Eltern beraten und ihnen beistehen. Dazu gehören privat sowie behördlich eingerichtete Stellen, die Mütter und Väter bei der lokalen Suche nach Fachpersonen unterstützen ${ }^{3}$, ein zunehmend umfassendes Betreuungsangebot auf den Gebärstationen selbst sowie auch Selbsthilfe-Vereine, die sich im Laufe der letzten Jahre professionalisiert und ihre Dienstleistungen ausgebaut haben. ${ }^{4}$ Hinzu kommt mehr und mehr Ratgeberliteratur. Diese Angebote tragen zur Communitybildung von Müttern und Vätern bei, die Eltern tot geborener Kinder sind. Sie ermöglichen den Austausch unter Menschen, die gleiches Leid erlebt haben und erleben. Flankierend präsentieren Psychologie, Soziologie und Pflegeforschung einzelne Studien, die auf Gesprächen mit betroffenen Eltern basieren ${ }^{5}$ oder den Umgang mit tot geborenen Kindern im medizinischen Alltag befragen. ${ }^{6}$ Analysiert werden auch Krisen des Trauerns ${ }^{7}$ sowie Rituale, die zur Bewältigung des Verlusts beitragen können. ${ }^{8}$

Ziel der verschiedenen Einrichtungen und Forschungsbeiträge ist es, die allgemeine Diskurs- und Wissensbildung über tot geborene Kinder zu fördern, um eine entsprechende Enttabuisierung und Aufklärung voranzutreiben. Dabei nun spielen insbesondere auch autobiografisch-künstlerische

3 Z. B. die Fachstelle Kindsverlust, www.kindsverlust.ch, die direkte Beratungen sowie Kontakte zu Fachpersonen anbietet. Informationen zu Fehl- und Totgeburt präsentiert etwa auch das Online-Portal der deutschen Bundeszentrale für gesundheitliche Aufklärung, www. familienplanung.de (letzter Zugriff jeweils: 23.11.2021).

4 Z. B. www.verein-regenbogen.ch; http://schmetterlingskinder.de; www.verein-pusteblume.at (letzter Zugriff jeweils: 23.11.2021).

5 Siehe z. B. Murphy (2010): Lost Futures oder Martin Kuse-Isingschulte: Psychische Belastungen durch eine Totgeburt und die Bewältigung. Verlauf, Einflussfaktoren, Stand und Möglichkeiten der therapeutischen Versorgung. Regensburg 2000.

6 Petra Rogge: „Knowing how? (Medizin)Ethische Fragen an den Umgang mit Stillgeburten im medizinischen Alltag“. In: Sic et Non. Zeitschrift für Philosophie und Kultur. Im Netz 9/1 (2008). Online unter: https://kops.uni-konstanz.de/bitstream/handle/123456789/55725/ Rogge_2-u8883gu2bxgy1.pdf?sequence=1\&isAllowed=y (letzter Zugriff: 23.11.2021).

7 Julia Böcker: „Kein Tod ohne Leben. Zu Krisen des Trauerns nach Fehl- und Totgeburten“. In: Stephan Lessenich (Hg.): Routinen der Krise - Krise der Routinen. Verhandlungen des 37. Kongresses der Deutschen Gesellschaft für Soziologie in Trier. Trier 2014.

8 Laurie Faro: „Postponed Ritual at the Site of a Monument: The Case of Stillborn Children“. In: Paul Post, Martin Hoondert (Hg.): Absent Ritual. Exploring the Ambivalence and Dynamics of Ritual. Durham, NC, 2019; Ying-Fen Tseng: „The Meaning of Rituals after a Stillbirth: A Qualitative Study of Mothers with a Stillborn Baby“. In: Journal of Clinical Nursing 27/5-6 (2018), S. 1134-1142. 
Darstellungen in verschiedenen Medien eine zentrale Rolle. Neben Autobiografien von Prominenten wie den eingangs erwähnten, die das Thema unter anderen behandeln, gibt es, wie im Folgenden anhand ausgewählter Beispiele gezeigt wird, autobiografische Bücher und Filme, die sich auf zentrale Weise mit Totgeburten befassen. Wenn Mütter - vereinzelt auch Väter - von tot geborenen Kindern ihre eigenen Erfahrungen gestalten, dann bringen sie dabei oftmals Wahrnehmungen und Befindlichkeiten zum Ausdruck, die weder in der Forschung noch in didaktisch-aufklärerischen Fachinformationen zur Sprache kommen. Schließlich gewinnt das Thema seit einem guten Jahrzehnt durch das Internet an Kontur und Sichtbarkeit: Hier veröffentlichen betroffene Eltern Bilder von ihren toten Babys, und zahllose Blogs und Foren ermöglichen es ihnen, miteinander in Kontakt zu treten und ihre Geschichte und Trauer öffentlich zu machen.

\section{Historische Vorläufer: Ingmar Bergman und William Kotzwinkle}

Heutige autobiografisch-künstlerische Darstellungen zu tot geborenen Kindern stehen eindeutig im Dienst einer themenbezogenen Diskursbildung und damit im Kontext gesellschaftlicher Aufklärungs- und Enttabuisierungsbemühungen. ${ }^{9}$

Frühere Darstellungen in Film und Literatur verfügten noch nicht über diesen Kontext. Sie blieben ähnlich isoliert wie die frühen Beispiele autobiografischer Sterbeliteratur aus den 1970er- bis 199oer-Jahren. Exemplarisch hierfür sind der in Schwarz-Weiß gedrehte Film Nära livet aus dem Jahr 1958 (dt. Nahe dem Leben) von Ingmar Bergman (1918-2007) sowie die Erzählung $A$ Swimmer in the Secret Sea (1975) von William Kotzwinkle (geb. 1943).

Bergmans Film spielt ausschließlich in den Innenräumen einer Entbindungsstation und porträtiert das Schicksal dreier Frauen, die ein Krankenzimmer teilen: Cecilia erleidet kurz nach ihrer Einlieferung eine Fehlgeburt; Hjördis hat erfolglos versucht, eine Abtreibung ihres ungewollten Kindes selbst herbeizuführen; das Kind der hochschwangeren Stina wird am Ende tot geboren. Das Drama kreist um die Fehlgeburt und die ungewollte Schwangerschaft, es thematisiert die schwierigen Beziehungsgeschichten von Cecilia und

9 Nicht behandelt werden hier literarische Darstellungen, die den Terminus „Totgeburt“ oder „Stillborn/Stillbirth“ poetologisch oder metaphorisch einsetzen. Beispiele hierfür sind etwa das Poem „Stillborn“ (1971, dt. „Tot geboren“) von Sylvia Plath, in dem die Autorin das Gedicht selbst als eine tot geborene Figur verhandelt, oder die Romane Das Erlernen der Totgeburt (1979) von Maria Erlenberger und stillborn (2014) von Michael Stavarič, welche psychische Grenzzustände vorführen und die Titelbegriffe im übertragenen Sinne für lebensverhindernde Haltungen der jeweiligen Protagonistinnen verwenden. 
Hjördis zu ihren Partnern und zeigt am Schluss Lösungsstrategien auf: Cecilia überdenkt die von ihr beschlossene Trennung von ihrem Mann, und Hjördis ringt sich dazu durch, ihre Mutter anzurufen, die sie nach der Entlassung aus dem Krankenhaus bei sich aufnimmt. Mit diesen beiden Fällen kontrastiert Stinas Schicksal. Ihre Schwangerschaft ist als einzige intakt und voller Glück: Gemeinsam mit ihrem Ehemann freut sie sich überschwänglich auf ihr Kind. Als die Wehen einsetzen, wird sie in den Kreißsaal gebracht, wo die Hebamme den Herzschlag des Kindes nicht mehr feststellen kann. Dieser Moment findet sich in verschiedensten filmischen und autobiografischen Erzählungen von tot geborenen Kindern, und er markiert stets einen klassischen Wendepunkt: Die Szene, in der die Herztöne des Babys nicht mehr auffindbar sind, kündigt die kommende Katastrophe an. In Bergmans Film holt die Hebamme den Arzt herbei, der ebenfalls keine Herztöne mehr vernimmt. Ungerührt gibt er Anweisung, Stina zu sedieren, und greift zur Zange. Die Geburt selbst wird filmisch ausgespart.

An nächsten Morgen liegt Stina, als Mutter eines nunmehr toten Kindes, wieder im Krankenzimmer bei Hjördis und Cecilia. Als der Arzt mit seiner mehrköpfigen Entourage zur täglichen Visite erscheint, geht er an ihrem Bett vorbei, ohne das Wort an sie zu richten. Sie ruft ihm hinterher, sie will wissen, warum das Baby tot ist. Er dreht sich zu ihr um, spricht ein paar wenige trockene Sätze: „As far as I can see there was nothing wrong with the boy. But ... - it wasn't meant to be." Er versichert ihr noch, dass es beim nächsten Mal durchaus klappen könne, und wendet sich wieder ab (vgl. Abb. 35 und 36 ).

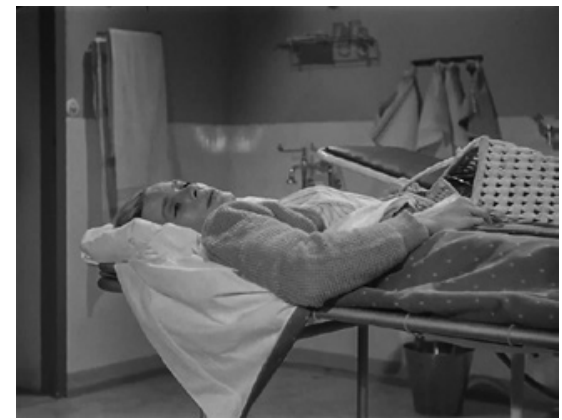

Abb. 35

Nära livet. Reg. Ingmar Bergman. Darst. Ingrid Thulin, Eva Dahlbeck, Bibi Andersson, Barbro Hior af Ornäs, Erland Josephson, Max von Sydow, Inga Leandgré. Schweden, Stockholm, Folkets Hus och Parker 1958. VHS. oo:04:35.

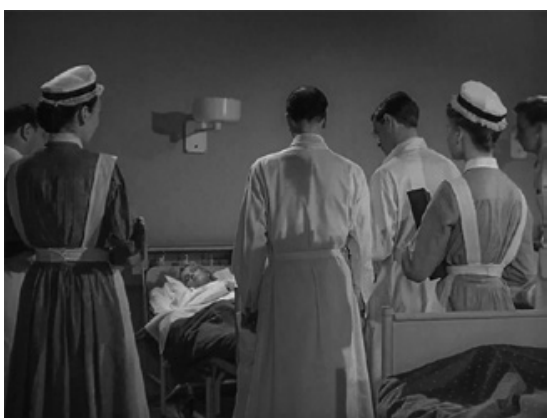

Abb. 36

Nära livet. Reg. Ingmar Bergman. Darst. Ingrid Thulin, Eva Dahlbeck, Bibi Andersson, Barbro Hior af Ornäs, Erland Josephson, Max von Sydow, Inga Leandgré. Schweden, Stockholm, Folkets Hus och Parker 1958. Film. o1:12:59. 
Im Gegensatz zur Fehlgeburt und zur ungewollten Schwangerschaft inszeniert Bergman das tot geborene Kind als eklatante Leerstelle. Es kommt nicht ins Bild, und es gibt keine aktive sprachliche Auseinandersetzung mit dem Problem des Verlusts, weder aus der Perspektive des Arztes noch aus jener der Patientin. Es findet keinerlei weitere Nach-Besprechung statt.

Demgegenüber kreist Kotzwinkles autobiografisch motivierte Erzählung, die seit der Erstpublikation im Jahr 1975 verschiedentlich neu aufgelegt wurde, in zentraler Weise um ein tot geborenes Kind. Ein personaler Erzähler namens Laski schildert hier das Geschehen aus der Sicht des Vaters, wobei die Zeitspanne wenige Tage vor, während und nach der Geburt umfasst. Die Erzählung beginnt mit dem Ruf der schwangeren Frau, dass die Fruchtblase geplatzt sei. Es ist Winter, eiskalt, die Szene spielt in Maine im Nordosten der USA; das Paar eilt mit dem Truck in die Klinik. Im Weiteren folgt eine seitenlange Schilderung der Geburt. Dies stellt bis heute eine große Rarität dar, figuriert doch die sprachliche Darstellung von Geburten nur selten in der Belletristik. ${ }^{10}$ Kotzwinkle aber beschreibt nüchtern und detailgenau den stundenlangen Verlauf der Wehen, das Kommen und Gehen des Pflegepersonals und des Arztes, das Warten, die Diskussionen über die Steißlage des Kindes, die vom Arzt eingeleitete Blasenentleerung.

Das Kind kommt mit den Füßchen voran zur Welt. Als es nach langer Zeit endlich ganz draußen ist, gibt es keinen Ton von sich, alles bleibt ruhig. Sofortige Beatmungsversuche scheitern, der Junge lebt nicht, schließlich gibt der Arzt auf: „He stepped back, wiped his brow, turned to Laski, and shook his head from side to side. Laski nodded. It was over."11

Am nächsten Morgen fährt Laski, ein Bildhauer, zurück nach Hause und zimmert aus Föhrenholz einen kleinen Sarg. Als er wieder ins Krankenhaus kommt, fragt ihn dort jemand auf dem Weg zur Entbindungsstation, was er denn bekommen habe („What did you have?"): „Laski hesitated as fragments of explanations rose in his mind - the baby died, we had nothing - but then he felt the spirit of the child again, suddenly surging in his heart, and he said, ,A boy. - ,Congratulations.“"12

Wenig später kann das Paar das Krankenhaus gemeinsam verlassen. Am Empfang erhält es den toten verhüllten Sohn überreicht. Der Vater nimmt

10 Vgl. dazu Stefanie Stockhorst: „Schwangerschaft und Geburt in der ,schönen“ Literatur. Überlegungen zum Funktionswandel eines Motivs". In:,Wenn sie das Wort Ich gebraucht". Festschrift für Barbara Becker-Cantarino von FreundInnen, SchülerInnen und KollegInnen. Amsterdam 2013, S. 41-71.

11 William Kotzwinkle (2010/1975): Swimmer in the Secret Sea. A Novella. Nottingham (2010/1975), S. $38 \mathrm{f}$.

12 Ebd., S. 47f. 
das Baby in den Arm, trägt es zum Auto, legt es in den Sarg und verschließt diesen mit dem Deckel. Den Sarg im Truck zwischen sich, fährt das Paar heim. Zuhause angekommen, nehmen sie ihn ins Haus und stellen ihn im Wohnzimmer hin. Abends sitzen sie schweigend da, und schließlich sagt die Mutter, dass sie ihren Sohn sehen möchte. Also öffnen sie den Sarg, enthüllen den Körper, der nunmehr grau und kalt ist, und stellen fest, dass ihm bei der Autopsie, die keinerlei Resultate erbracht hat, sämtliche Organe entnommen wurden. Tags darauf begräbt Laski den toten Sohn mithilfe eines Freundes.

Im Gegensatz zu der heutigen Aufbereitung des Themas fehlt bei dieser Erzählung jegliche didaktische Stoßrichtung, gerade dies zeichnet den künstlerischen Ansatz aus. Beispielhaft deutlich wird das unter anderem, wenn der Vater vor dem geöffneten Sarg steht:

He looked at the face of his son and saw that it had undergone a strange transformation. The features had completely matured, the face now that of a man of many years, as if the single moment of life when he was spun upon the doctor's hand had been a lifetime from beginning to end. ${ }^{13}$

Hier geht es nicht primär darum vorzuführen, wie wichtig der Anblick des toten Kindes für die spätere Trauerverarbeitung ist. Vielmehr kommt zur Sprache, was der Anblick selbst entbirgt. Darin manifestiert sich ein poetischreflektiver Zugang, der in gewisser Weise zeitlos ist und nicht zu einer gesellschaftlichen Diskursbildung und Enttabuisierung beizutragen sucht, wie es bei heutigen Projekten zu tot geborenen Kindern in Film und Literatur der Fall ist. Kotzwinkles eindringliche Literarisierung der Totgeburt steht außerhalb eines didaktisch-aufklärerischen Rahmens und figuriert damit bis heute als poetischer Sonderfall.

\section{Heutiger Aufklärungs- und Enttabuisierungsdiskurs: Frauke Kohl, Alice Jolly, Tina Brenneisen, Sean Hanish}

Einer der ersten expliziten Beiträge zur Enttabuisierung tot geborener Kinder im deutschsprachigen Raum ist die autobiografische Geschichte einer Totgeburt. Henriette von Dessau (2008) von Frauke Kohl. Die Autorin schildert die Geburt ihrer toten Tochter Henriette, welche 1985 in einem Krankenhaus in Dessau stattfand. Es handelt sich um einen Erfahrungsbericht, veröffentlicht mit dem Ziel, zu einem neuen medizinisch-gesellschaftlichen Umgang mit tot

13 Ebd., S. 77. 
geborenen Kindern beizutragen. Analog zu Bergmans Film ist auch diesem Bericht zu entnehmen, dass es im Krankenhaus zu keinerlei Gesprächen über das tot geborene Kind gekommen ist, und wie in Nära livet wird auch hier der Mutter jeglicher Blick auf das tote Kind verwehrt.

Kohl ist im Dezember 1985 zur Entbindung im Krankenhaus. Auch in diesem Fall ist die Szene mit dem Holzhörrohr der Wendepunkt: Bei einer morgendlichen Routineuntersuchung kann die Hebamme den Herzschlag des Kindes nicht mehr feststellen. Am frühen Nachmittag erfolgt eine weitere Untersuchung durch einen Arzt mit einem Ultraschallgerät, das Resultat ist dasselbe. Nach der Todesbotschaft geht Kohl auf ihr Zimmer, wo sie bis abends warten muss, bevor die Geburt eingeleitet wird, denn „so etwas müssen wir nachts machen, wenn der [Kreiß]Saal leer ist. ${ }^{\text {"4 }}$ Die Geburtsleitung wird einer jungen, unerfahrenen Hebamme übertragen, die hier üben soll, wie man Kinder zur Welt bringt. Fast zehn Mitglieder des Personals finden sich ein, um dem seltenen Schauspiel zuzusehen. Frauke Kohl ist während der Geburt ohne Beistand, ihr Mann und ihr Schwiegervater wurden vom Personal abgewiesen. Als das Kind kommt, hält ihr eine weitere Hebamme ein OP-Tuch vor der Brust hoch, um ihr die Sicht auf ihr totes Baby zu verwehren. Dieses wird in einer Aluminiumschüssel weggebracht, die Mutter bekommt es nie zu sehen. Ein paar Tage später kann sie das Krankenhaus verlassen, ihr Mann und ihr Vater holen sie ab, sie gehen ohne ein Kind nach Hause.

Der Bericht, über zwanzig Jahre nach der Geburt veröffentlicht, fungiert einerseits als Anklage gegen diejenigen, die der Mutter damals das tote Kind vorenthielten, und andererseits als starkes Plädoyer für würdevolles Abschiednehmen. Die Autorin hält fest, wie wichtig es für eine Mutter sei, was ihr selbst versagt blieb: Das Kind im Arm zu halten, „auch das tote Kind, gerade das tote

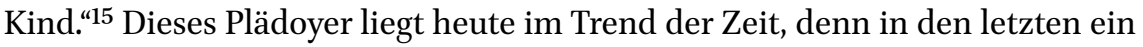
bis zwei Jahrzehnten fand ein deutlicher Paradigmenwechsel statt: Es gilt, dass Mütter ihre toten Kinder anschauen und von ihnen Abschied nehmen können sollen.

Ein eindringliches Beispiel dafür gibt die britische Autorin Alice Jolly (geb. 1966) in ihrem preisgekrönten autobiografischen Buch Dead Babies and Seaside Towns (2017). Nachdem im sechsten Schwangerschaftsmonat diagnostiziert wurde, dass ihr Kind nicht überlebensfähig sein würde, bringt die Ich-Erzählerin es auf natürlichem Wege zur Welt. Wie von Kohl gefordert, kommt es dabei zweimal zur Anschauung des toten Kindes.

Die erste Anschauung erfolgt direkt bei der Geburt:

14 Frauke Kohl: Geschichte einer Totgeburt. Henriette von Dessau. Gelnhausen 2008, S. 5 ○.

15 Ebd., S. 97. 
After all this time, I'm excited to see her. And what do I see? Some knot of cells, some object less than human? No. What I see is someone who is more fully human than anyone I've ever seen before. [...] She is dark red, bony, beautiful. Her soul is visible under her skin. Her face is feline, eternal. It has the serenity of an Egyptian mask. Every detail is complete - fingers, toes, eyebrows, the perfect globe of her head, marked by a mesh of veins, the fan of her tiny ribs. She's warm in my arms and surely only sleeping? ${ }^{16}$

Nachdem sich die Mutter von der Geburt ausgeruht hat, wird der kleine Leichnam in einem Kinderbettchen nochmals zu ihr gebracht, so dass sie ihn ein zweites Mal anschauen kann. Diese Beschreibung tendiert dabei, ähnlich wie bei Kotzwinkle, zum Analytisch-Deskriptiven:

A flower has been placed next to her head. Her skin is dark now, almost black, and she looks stiff. Her flesh is shrinking so that her skull shows through behind her face. Her bottom jaw is sagging, the lower part of her face caving it. I feel as though I should pick her up but I'm frightened. She looks so brittle she might break. $^{17}$

Im Weiteren nehmen die Ich-Erzählerin und ihr Mann das tote Baby, analog der Erzählung von Kotzwinkle, in einem kleinen Sarg nach Hause, wo sie sich dazu entscheiden, diesen auf den Esstisch zu stellen. Am nächsten Tag transportieren sie ihn mit dem Eurostar-Zug von Brüssel nach England - der Sarg muss durch den Scanner -, um Laura dort im Beisein der Familie zu bestatten.

Jollys Buch setzt ausführlich und konkret in Szene, was heute allgemein bei tot geborenen Kindern empfohlen wird: sich Zeit und Raum für die Verabschiedung zu nehmen und dies nach Möglichkeit aktiv zu gestalten. Hierzu gehört auch, dass die Entbindungsstationen spezielle Räume für tot geborene Kinder und ihre Eltern bereitstellen und dass sie außerdem Utensilien wie Tücher, Blumen und Kerzen verfügbar halten. Bisweilen wird auch, wie von Kotzwinkle und Jolly vorgeführt, explizit die Möglichkeit angesprochen, das verstorbene Kind mit nach Hause zu nehmen, um sich von ihm in denjenigen Räumen zu verabschieden, in welchen es gelebt hätte. ${ }^{18}$

Ebenfalls nahegelegt wird die Aufhebung von Erinnerungsobjekten (z. B. ein Fußabdruck oder eine Decke, in die das Kind gehüllt war). Im Weiteren unerlässlich und gängig ist heute die Namensgebung, die wesentlich zur Annahme eines toten Kindes beiträgt. Auch diesen Punkt spricht Frauke

\footnotetext{
16 Alice Jolly: Dead Babies and Seaside Towns. London 2017, S. 37.

17 Ebd., S. 42.

18 Heike Wolter: Mein Sternenkind. Begleitbuch für Eltern, Angehörige und Fachpersonen nach Fehlgeburt, stiller Geburt oder Neugeborenentod. Salzburg 2012, S. 146.
} 
Kohl in ihrem Bericht offensiv an, denn bei ihrer tot geborenen Tochter wurde noch nicht einmal nach einem Namen gefragt. In der Sterbeurkunde hieß es schlicht: „ein Kind [...] tot geboren. ${ }^{19}$ Bei Jolly hingegen wird der Name just in dem Moment zum Thema, als klar wird, dass das Kind die Geburt nicht überleben wird: „I want her to have a name [...]. I like the name Laura. ${ }^{“ 20}$

Besonders gefördert wird heute zudem die Körpererfahrung. So sollen Eltern ihr totes Baby in den Arm nehmen, es allenfalls waschen und anziehen, um es als das ihrige anzuerkennen. Franziska Maurer, Mitbegründerin und langjährige Leiterin der Berner Fachstelle Fehlgeburt und perinataler Kindstod, sagt hierzu:

Es [das tote Baby] anschauen, in den Arm nehmen, zu Hause ins vorbereitete Bettchen legen - all dies schafft eine gemeinsame Geschichte mit dem verstorbenen Baby. Wichtig ist, dass es seinen ganz eigenen Platz und den ihm gebührenden Respekt bekommt. Es kam, war da und ging wieder. Wie jeder Mensch. Die Zeit seines Bleibens war einfach sehr kurz. ${ }^{21}$

Allerdings stellt ein derart bewusster Umgang mit der Trauer viele Eltern vor schwere Herausforderungen, denn im ersten Schock bleibt vielfach kaum Raum dafür. Bei Jolly etwa macht sich in der zitierten Passage gerade die Unfähigkeit bemerkbar, das Kind in die Arme zu nehmen („I feel as though I should pick her up but I'm frightened.").

Zweifellos begünstigt die Möglichkeit Abschied zu nehmen eine nachfolgende psychische Bewältigung. Dazu gehört auch die Frage der Bestattung. Frauke Kohl berichtet, dass ihr Kind in Dessau kremiert und die Urne anschließend per Post zum Friedhof nach Jeßnitz geschickt wurde, wo eine Friedhofsgärtnerin sie stillschweigend ohne jegliche Zeremonie im Familiengrab beisetzte. Heute gibt es ein vielfältiges Angebot für die Bestattung tot geborener Kinder sowie auch, je nach Wunsch, für die Bestattung fehl geborener Kinder, für die keine Meldepflicht besteht. Immer mehr Friedhöfe bieten hierfür eigene Grabfelder oder spezielle Gemeinschaftsgräber an (vgl. Kap. 7, S. 124-125). Gedenkfeiern ermöglichen überdies auch den Einbezug des sozialen Umfelds, der den Abschied von dem toten Kind weiter festigt. ${ }^{22}$ Jolly beschreibt in ihrem Buch, wie die Familie den kleinen Sarg von Belgien nach Upton-upon-Severn im Süden Englands transportiert, zunächst zum

19 Kohl (2008): Geschichte einer Totgeburt, S. 140.

20 Jolly (2017): Dead Babies and Seaside Towns, S. 35.

21 Franziska Maurer: „Wenn Geburt und Tod zusammen fallen“. In: pflegen:palliativ 36 (2017), S. 19 .

22 Siehe dazu Tseng (2018): The Meaning of Rituals after a Stillbirth. 
Haus ihrer Mutter, dann in eine nahe gelegene Kirche, in der sie und ihr Ehemann vier Jahre zuvor geheiratet hatten. Nach einem Gottesdienst, an dem Freund:innen und die Familie teilnehmen, wird Laura auf dem Kirchenfriedhof begraben. Anschließend sitzt die Trauergemeinschaft im Haus der Mutter bei Tee und Whisky zusammen.

Im Zentrum der künstlerisch-autobiografischen Darstellungen steht die Bemühung, Worte und Bilder für den physischen und psychischen Schmerz und dessen langes Anhalten zu gestalten. Exemplarisch hierfür ist auch die 234 Seiten lange Graphic Novel Das Licht, das Schatten leert (2019), in der die Berliner Comiczeichnerin Tina Brenneisen (geb. 1977) ihre Erfahrung als Mutter eines tot geborenen Sohnes, Lasse, zur Darstellung bringt. Für den Band erhielt sie die höchstdotierte deutsche Comicauszeichnung, den Berthold-Leibinger-Preis.

Der Comic setzt in der Kühlhalle eines Krankenhauses ein: Ein Mitarbeiter entnimmt einer Schublade ein totes Baby und reicht es einer Pflegefachfrau, die damit im Aufzug zu den Krankenräumen fährt und es dort den Eltern übergibt. Was es heißt, mit solchem abrupten Einbruch des Todes konfrontiert zu sein, der an die Stelle der aufgeregten Vorstellung eines lebendigen Babys tritt, wird im Weiteren in mannigfachen Szenen dargestellt. Brenneisen schildert das Alleingelassen-Werden der Eltern, die Hilflosigkeit des familiären Umfeldes, soziale Isolation in der Nachbarschaft, Beginn einer Therapie sowie Gespräche über eine weitere Schwangerschaft.

Der Comic zeichnet sich durch eine komplexe erzählerische und gestalterische Dramaturgie aus. Die chronologische Erzählung wird wiederholt durch Rückblenden, Träume und negative emotionale Höhepunkte unterbrochen, die sich farblich von der Haupterzählung abheben: Der Alltag, in den sich die Eltern Tini und Fritzemann nach der traumatischen Erfahrung zurückziehen, ist dominiert von gräulich-grünlichen Farbtönen, welche die dumpfe Gleichförmigkeit des seelischen Erlebens symbolisieren. Damit kontrastieren expressive Farben, wenn die akute physische und psychische Schmerzerfahrung zur Darstellung kommt. Ein Beispiel hierfür ist das traumatische Wiedererleben der Geburt (vgl. Abb. 37).

Eine andere Szene zeigt die zunehmende Verlorenheit des trauernden Elternpaares (vgl. Abb. 38).

So schildert das Buch detailliert und ausführlich die Wucht, mit welcher der Tod Lasses über das Paar hereinbricht und danach anhaltend präsent bleibt. Insbesondere das Befinden der Mutter bleibt über Jahre hinweg instabil, da sie keine ausreichende familiäre Unterstützung erhält und an schweren Schuldgefühlen leidet. Die Stärke des Comics liegt in der bildlichen Darstellung des Unausgesprochenen, Unaussprechlichen. Das traumatisierende Erlebnis 

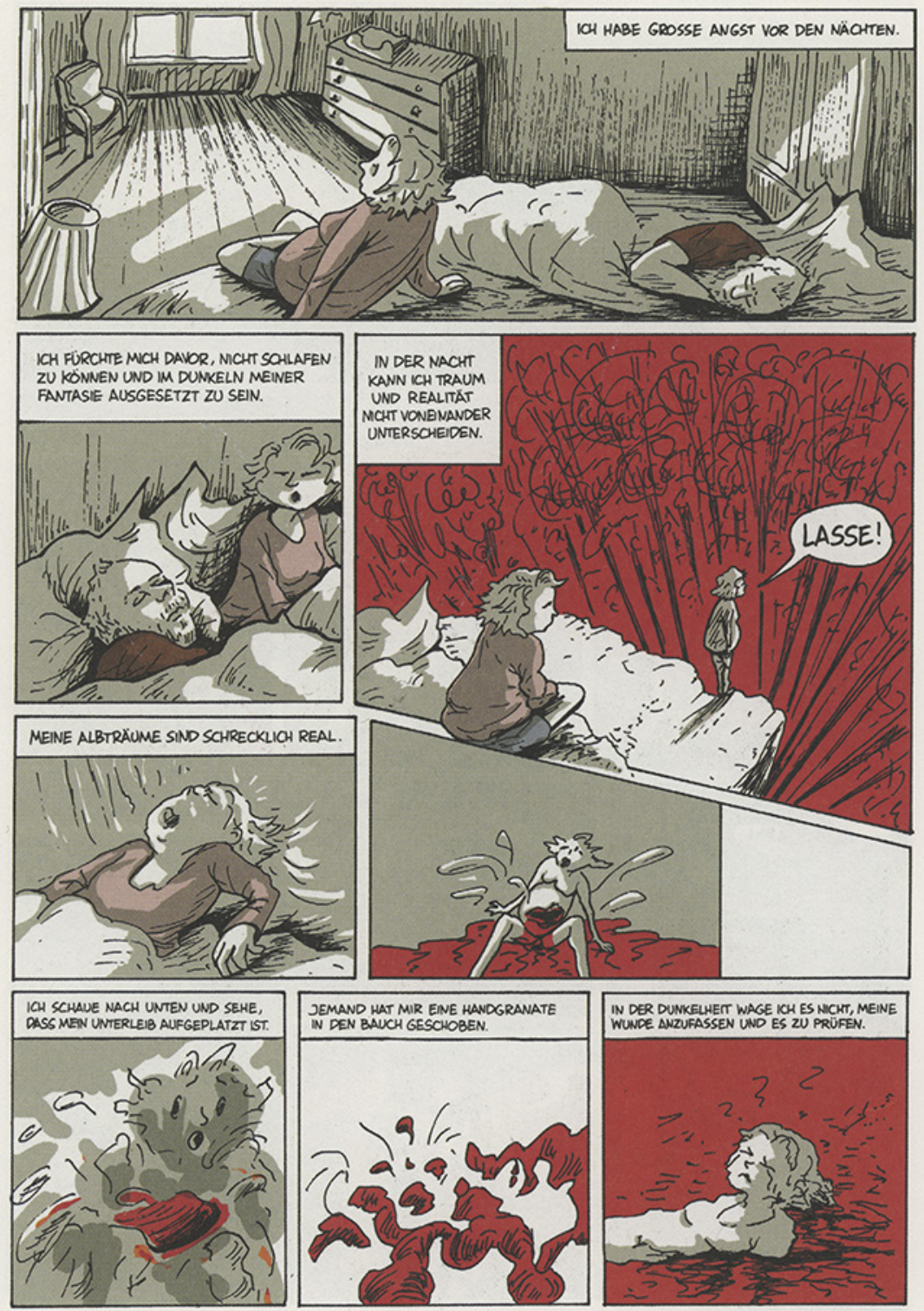


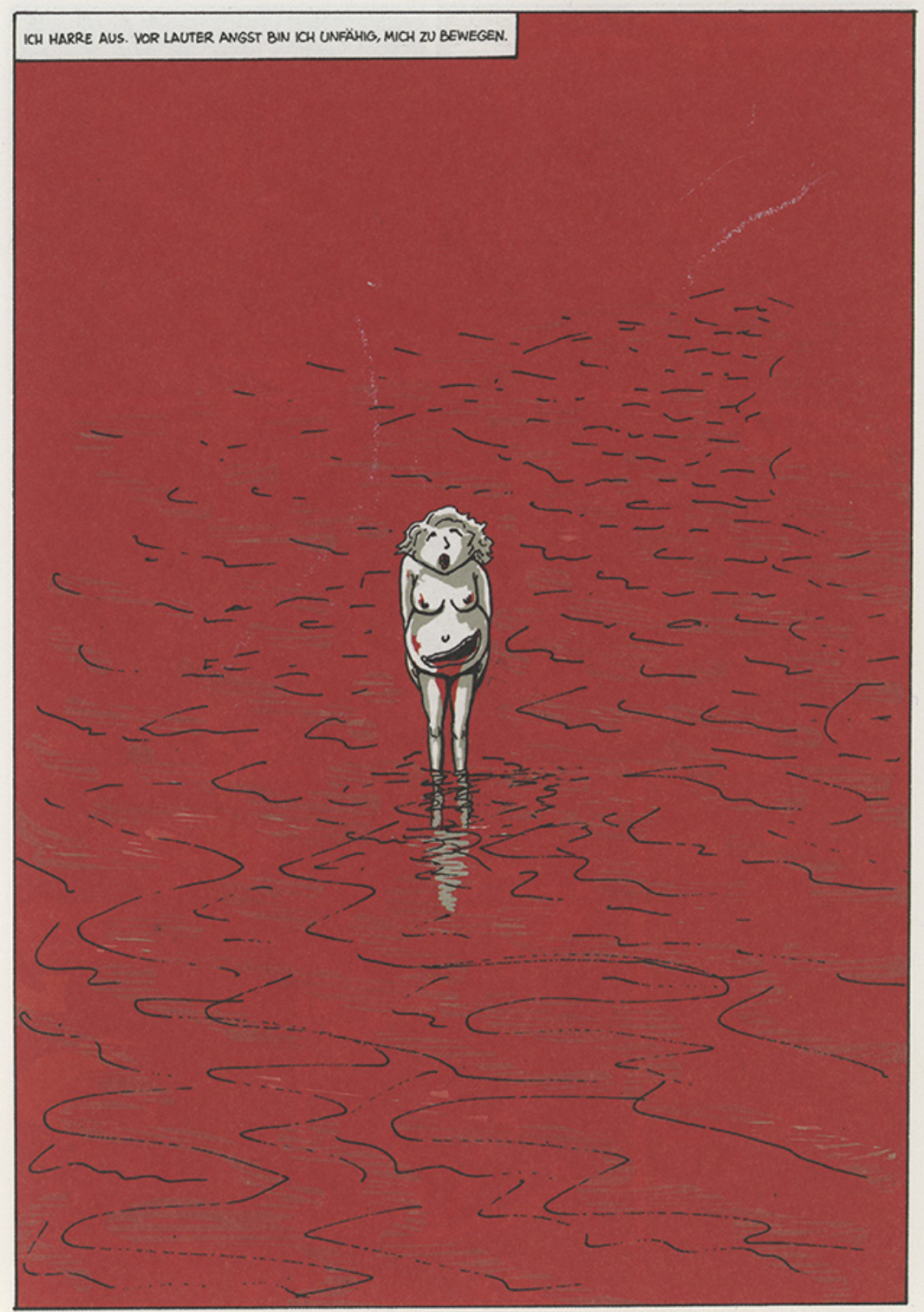

Abb. 37 Tina Brenneisen: Das Licht, das Schatten leert. Zürich 2019, S. 20-21. Mit freundlicher Genehmigung der Künstlerin. 

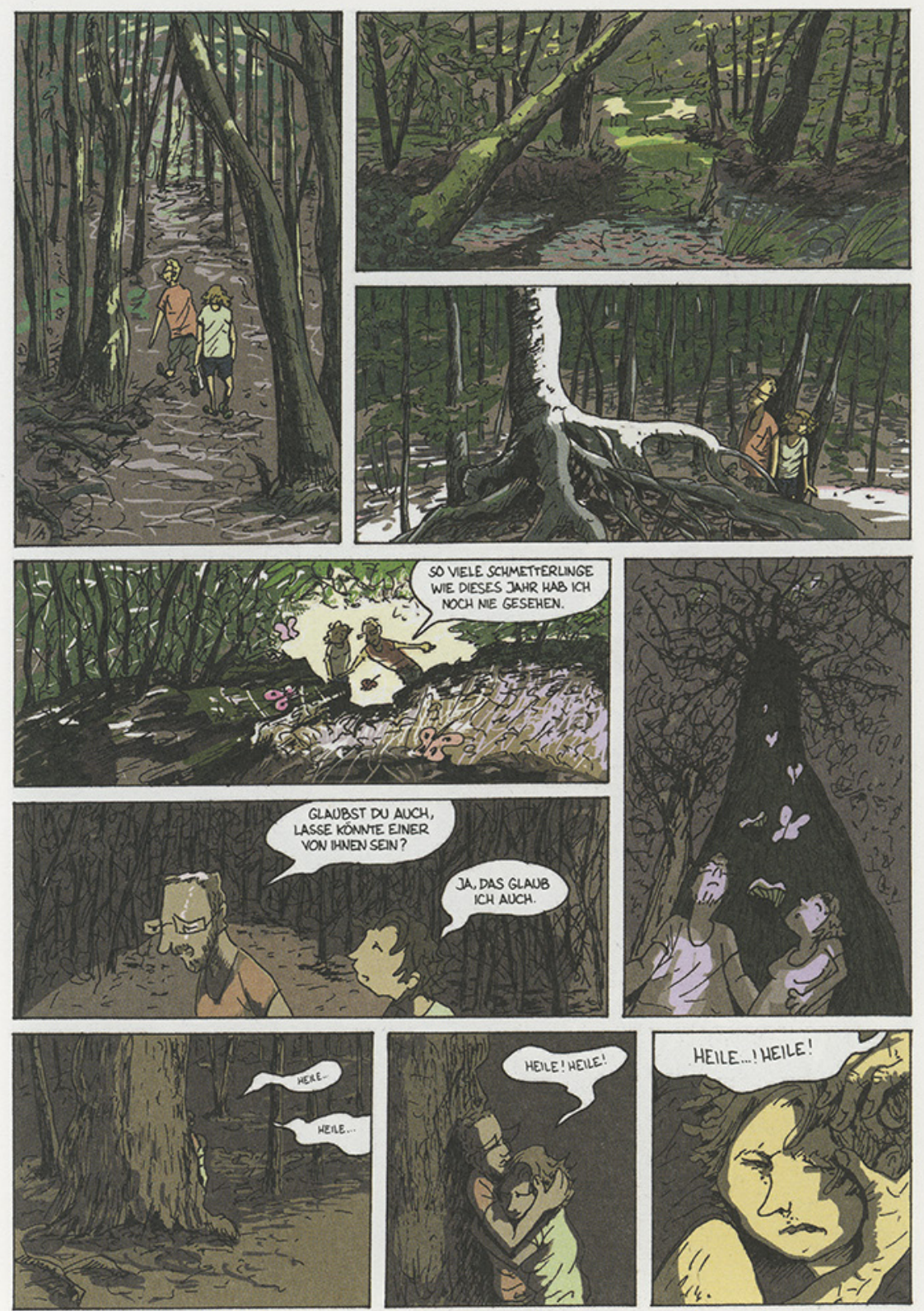

Abb. 38

Tina Brenneisen: Das Licht, das Schatten leert. Zürich 2019, S. 91. Mit freundlicher Genehmigung der Künstlerin. 
schlägt sich zeichnerisch nieder in deformierten Körpern und unförmigen, schmerzhaft ins Fratzenhafte verzogenen Gesichtsausdrücken. Wiederholt gelingen der Autorin eindringliche Bildsequenzen für die abgrundtiefe Leerstelle, die das tote Kind hinterlassen hat, wie etwa die Zeichnung des mütterlichen Körpers als Sarg (vgl. Abb. 39).

In einer träumerischen Sequenz, in der die Mutter das Kleinkind auf den Schultern trägt, klaffen schwarze Löcher anstelle des Gesichts, der Händchen und Füßchen (vgl. Abb. 40).

Gerade die langatmige, ausharrende und insistierende Gestaltung der Geschichte gehört zu den Stärken des Comics. Am Ende präsentiert er, wie etwa auch Jollys Autobiografie, eine Kontaktliste mit Angaben zu institutionellen Einrichtungen, die verwaisten Eltern in ihrem Schmerz beistehen.

Als exemplarisch für Filme, die tot geborene Kinder zwecks Aufklärung und Enttabuisierung in Szene setzen, kann Return to Zero (2014) des US-Amerikaners

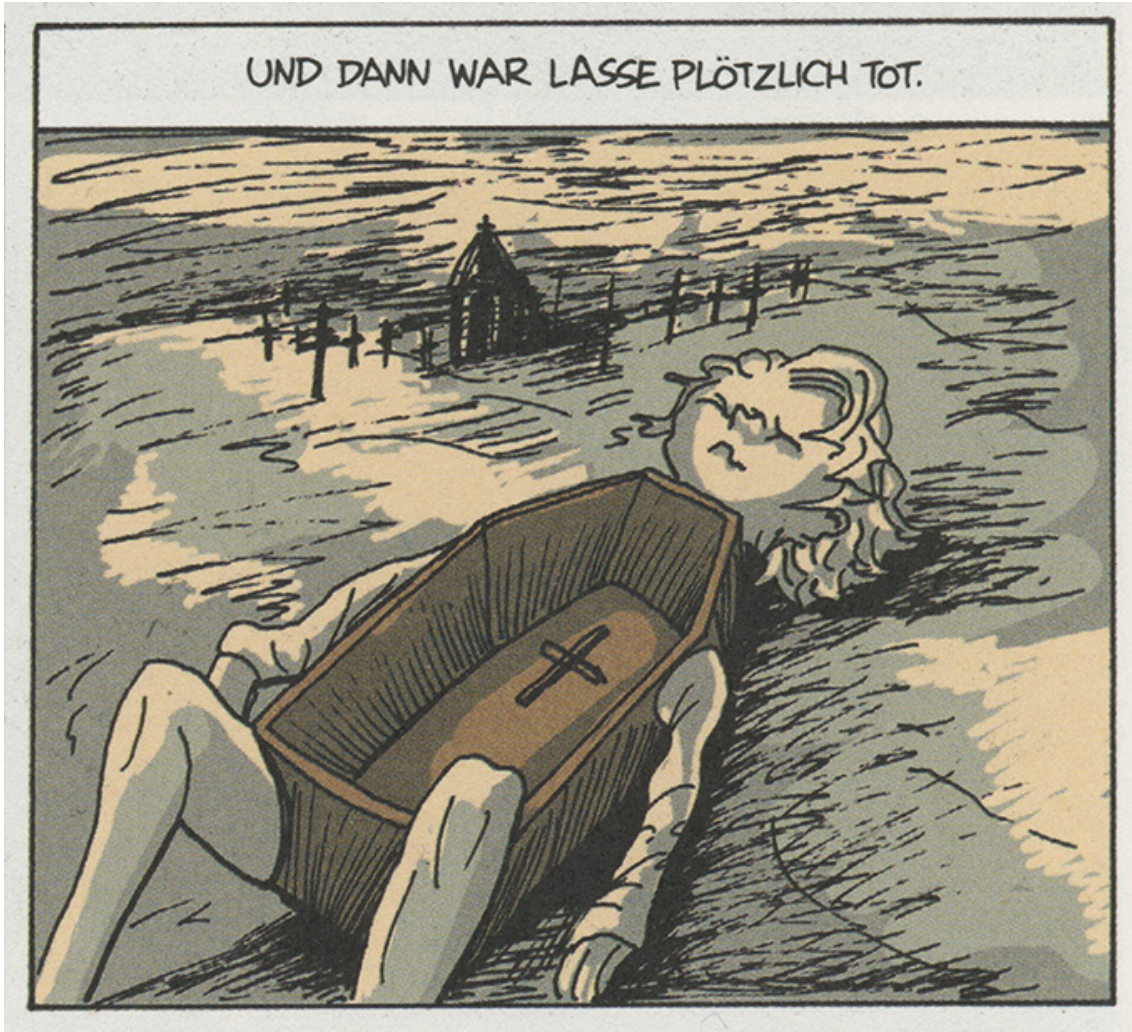

Abb. 39 Tina Brenneisen: Das Licht, das Schatten leert. Zürich 2019, S. 17. Mit freundlicher Genehmigung der Künstlerin. 


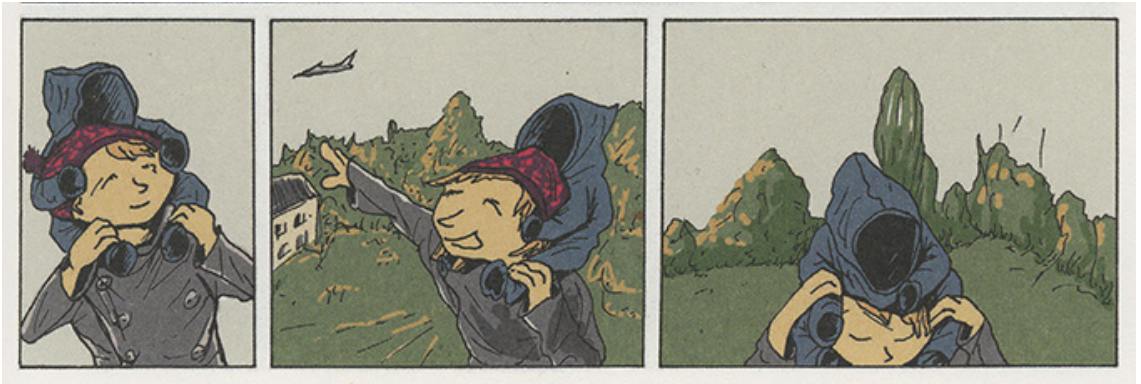

Abb. 40 Tina Brenneisen: Das Licht, das Schatten leert. Zürich 2019, S. 215. Mit freundlicher Genehmigung der Künstlerin.

Sean Hanish gelten. „Based on a true story“, ist der Spielfilm dem 2005 tot geborenen Sohn Hanishs gewidmet. Die Finanzierung kam unter anderem durch Beiträge von über 700 Familien zustande, die selbst ein "stillborn baby“ zu beklagen haben - um die gleiche Zeit finanzierte auch Alice Jolly ihre Autobiografie über eine literarische Crowdfunding Plattform. So tritt der Beitrag der künstlerischen Arbeiten zur Community-Bildung, welche einen gemeinsamen Diskurs erfordert, in den Vordergrund.

Hanishs Film porträtiert ein erfolgreiches junges Paar, das sich auf sein erstes Kind freut, dargestellt anhand einer Reihe konventioneller Szenen aus dem Geburtsvorbereitungskurs, dem Berufsleben und Familienkreis sowie der Babyparty. Wie schon bei Bergman und Kohl markieren auch hier einige fast beiläufige Worte des Arztes bei einer Routineuntersuchung den dramatischen Umkehrpunkt: „I can't find the baby's heartbeat." Vor die Wahl zwischen Kaiserschnitt und natürliche Geburt gestellt, entscheidet sich die Mutter für die natürliche Geburt, doch deren filmische Darstellung bleibt vorerst ausgespart. Stattdessen wird der monatelange Verarbeitungskampf des Paares in Szene gesetzt, wobei jeder für sich allein mit dem Kindsverlust fertigzuwerden versucht, so dass Streit und Entfremdung dominieren. Die Beziehungskrise wird schließlich mit einer nächsten Schwangerschaft beendet; in diesem Moment erst wird die vorangehende Geburt des toten Babys in einer Rückblende gezeigt.

Das Motiv einer neuen Schwangerschaft taucht in den meisten autobiografischen Darstellungen auf. Es geht dabei aber mehrheitlich nicht um eine Ersetzung des tot geborenen Kindes durch ein nächstes oder darum, die traumatische Erfahrung mit einem lebendigen Kind zu verdecken oder zu bewältigen. Vielmehr scheint die Geburt eines weiteren Kindes das öffentliche Sprechen über das vorangegangene tot geborene Kind zu begünstigen. Zwischen der Totgeburt und den autobiografisch-künstlerischen Veröffentlichungen liegen im Regelfall mehrere Jahre, oft über ein Jahrzehnt. 
Eine andere Richtung schlägt der Ende 2020 erschienene und ebenfalls autobiografisch motivierte US-Film Pieces of a Woman von Kornél Mundruczó ein; die Drehbuchautorin Kata Webér, die Ehefrau des Regisseurs, hatte selbst eine Fehlgeburt erlitten. Auch in diesem Film geht es um ein Baby, das unmittelbar nach der Geburt stirbt, und um ein Paar, das den Verlust nicht gemeinsam auffangen kann. Im Fokus steht eine Anklage der Hebamme, welche die Mutter (Vanessa Kirby) schließlich zurücknimmt. Filmgeschichtlich außergewöhnlich und herausragend ist die eröffnende Darstellung einer ungeschnittenen 23-minütigen Geburtsszene. Im Anschluss jedoch werden Strategien der Trauerbewältigung, wie etwa die Abschiednahme von dem toten Körper oder das Ritual der Bestattung, nur flüchtig thematisiert; im Vordergrund steht bis zum Schluss die innere Versteinerung der Mutter und der Zerfall einer Ehe. Somit stellt der Film vor Augen, wohin der Verlust eines Babys führen kann, wenn man sich mit der Trauer nicht aktiv auseinandersetzt.

\section{Die Fotografie des toten Babys: Vom Film zum Internet}

Im Unterschied zu literarischen Bearbeitungen von Totgeburten und auch zum Comic kann der Film potenziell foto-realistische Bilder der kleinen Leichname zeigen. Return to Zero stellt sich dieser Herausforderung auf indirektem Weg, indem zunächst mehrmals hintereinander Fotografien des toten Jungen Arthur sowie deren Bedeutung für die Betroffenen präsentiert werden. So steht bei der Gedenkfeier am Ufer des Meeres, an der die ganze Familie teilnimmt, neben der Urne auch ein Foto des tot geborenen Kindes auf einem Tisch. Als die Mutter später zu einer Untersuchung zur Ärztin geht, enthüllt ihr diese, dass auch sie vor Jahren ein totes Baby geboren habe, und zeigt ihr ein entsprechendes Foto. Im Folgenden kommt es zwischen den Eltern von Arthur zu einem Streit wegen einer Affäre des Mannes; da legt ihm seine Frau, gleichsam als Mahnmal, ein Foto des gemeinsamen toten Babys hin. Zu guter Letzt wird das Baby bei der Rückblende der Geburt in langen Einstellungen ausführlich gezeigt: Die Mutter hält es an ihre Brust, der Vater nimmt es in die Arme, und zusätzlich werden auch noch einige Schwarz-Weiß-Fotografien eingeblendet, die die Eltern mit dem toten Baby im Arm zeigen und somit den dokumentierenden Akt des Fotografierens selbst festhalten (vgl. Abb. 41 bis 45$){ }^{23}$

23 Vgl. auch den auf YouTube veröffentlichten Kurzspielfilm The Deafening Silence - Stillbirth through a Mother's Eyes (2013): In der zentralen didaktisch-aufklärerischen Szene fragt die Hebamme die junge Mutter nach der Geburt: „Do you want to see your daughter?“ - „No, no", sagt diese und wendet sich ab. Da geht der Vater hin, nimmt das tote Mädchen in seine Arme und bringt es der Mutter, die sich ihm nach einiger Zeit doch noch zuwendet. Online unter: https://www.youtube.com/watch?v=MQolbL6Qcqo (letzter Zugriff: 23.11.2021). 
Hinter solch aktuellem Bilddiskurs bleibt der deutsche Spielfilm Am Himmel der Tag (2012) von Pola Beck deutlich zurück. Der Film setzt das unstete Leben der Architekturstudentin Lara in Szene, die schwanger wird und schließlich ein totes Kind zur Welt bringt. Zwar kommt der Abschied vom toten Kind ins Bild, wenn es in einem Korb zu Lara gebracht wird und sie es in den Arm nimmt. Doch das Gesicht des Babys bleibt dabei undeutlich und verschwommen, als gälte es eine Privatsphäre zu schützen oder als wäre ein allzu realistisches Bild eine Zumutung für die Zuschauerschaft (vgl. Abb. 46 und 47). Auch in Pieces of a Woman wird kein Bild des toten kleinen Leichnams gezeigt.

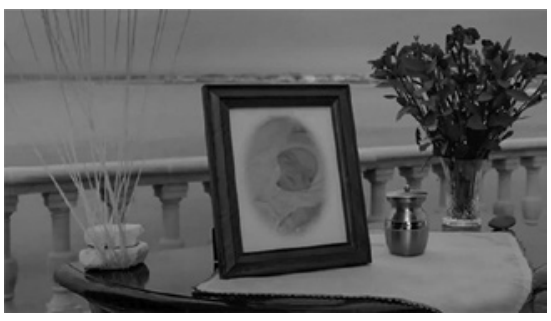

Abb. 41

Return to Zero. Reg. Sean Hanish. Darst.

Minnie Driver, Paul Adelstein, Alfred Molina, Connie Nielsen, Andres Anders, Kathy Baker, Sarah Jones. USA, Cannonball Productions 2014. Film. O०:23:04.

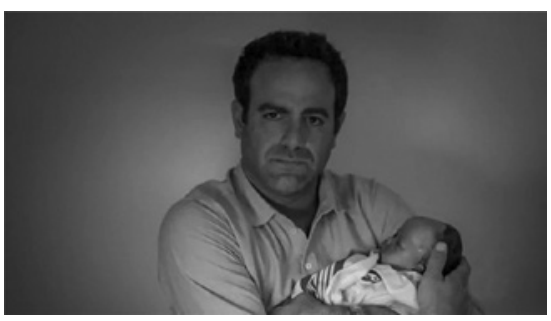

Abb. 43

Return to Zero. Reg. Sean Hanish. Darst. Minnie Driver, Paul Adelstein, Alfred Molina, Connie Nielsen, Andres Anders, Kathy Baker, Sarah Jones. USA, Cannonball Productions 2014. Film. o०:57:59.

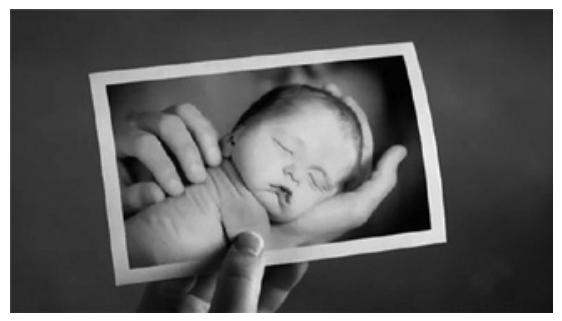

Abb. 42

Return to Zero. Reg. Sean Hanish. Darst.

Minnie Driver, Paul Adelstein, Alfred Molina, Connie Nielsen, Andres Anders, Kathy Baker, Sarah Jones. USA, Cannonball Productions 2014. Film. oo:52:32.

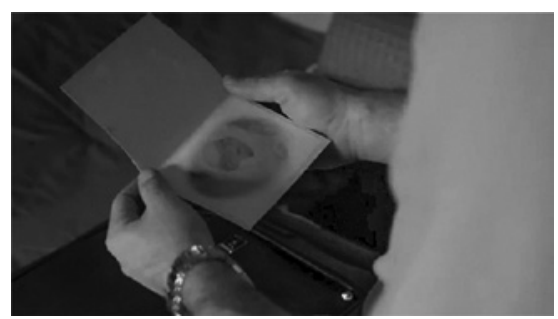

Abb. 44

Return to Zero. Reg. Sean Hanish. Darst. Minnie Driver, Paul Adelstein, Alfred Molina, Connie Nielsen, Andres Anders, Kathy Baker, Sarah Jones. USA, Cannonball Productions 2014. Film. 01:08:07. 
Abb. 45

Return to Zero. Reg. Sean Hanish. Darst. Minnie Driver, Paul Adelstein, Alfred Molina, Connie Nielsen, Andres Anders, Kathy Baker, Sarah Jones. USA, Cannonball Productions 2014. Film. 01:16:04.
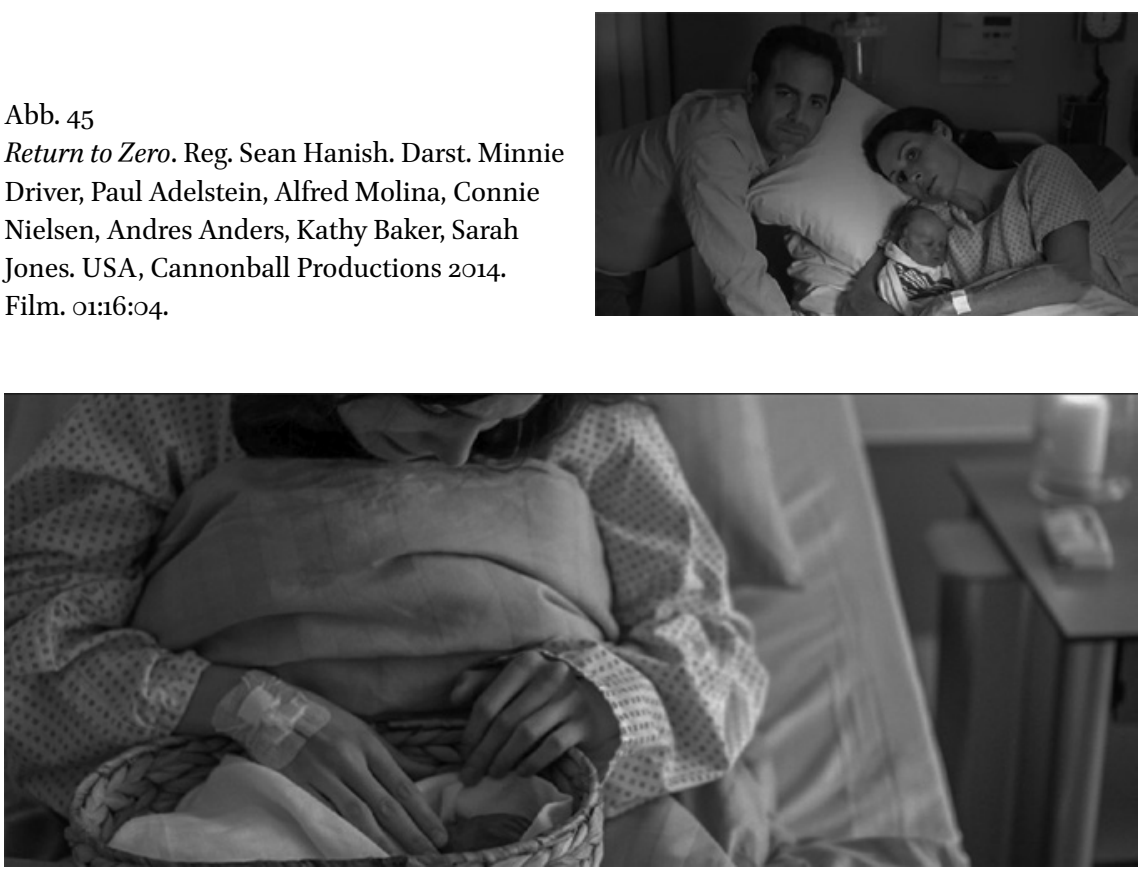

Abb. 46 Am Himmel der Tag. Reg. Pola Beck. Darst. Aylin Tezel, Henrike von Kuick, Tómas Lemarquis, Godehard Giese, Marion Mitterhammer, Lutz Blochberger, Anja Karmanski, Mai Michael Müller. Deutschland: ALIN Filmproduktion 2012. DVD. 01:10:49.

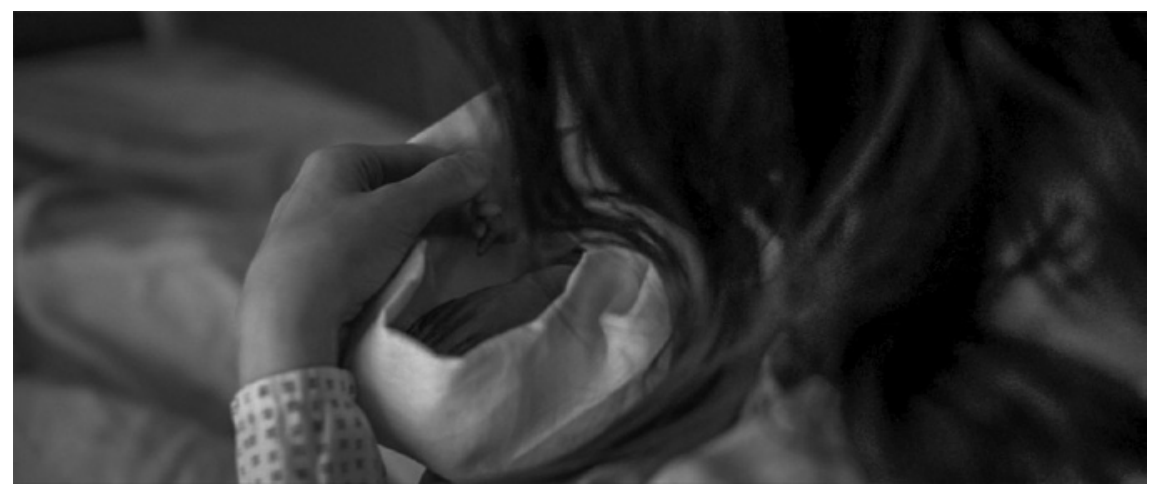

Abb. 47 Am Himmel der Tag. Reg. Pola Beck. Darst. Aylin Tezel, Henrike von Kuick, Tómas Lemarquis, Godehard Giese, Marion Mitterhammer, Lutz Blochberger, Anja Karmanski, Mai Michael Müller. Deutschland: ALIN Filmproduktion 2012. DVD. 01:11:21. 
Eine massive Zunahme hat die Verbreitung von fotografischen Bildern toter Babys durch das Internet erfahren. Trauernde Eltern können dort nicht nur fachliche Informationen abrufen und in Kontakt mit anderen Eltern treten, die das gleiche Schicksal erleiden, sondern das Internet ermöglicht es ihnen auch, Fotos ihrer tot geborenen Kinder zu veröffentlichen.

Virtuelle Friedhöfe verzeichnen gemeinhin vor allem unerwartete und daher besonders schwer zu bewältigende Todesfälle; neben tot geborenen Babys sind dies insbesondere Todesfälle von Kindern, Jugendlichen und jüngeren Personen, die an Krankheiten gestorben sind, einen Unfalltod erlitten oder Suizid verübt haben. Die entsprechende Trauer von Hinterbliebenen kann sich dabei auch entgegen geltender gesellschaftlicher Schweigegebote - besonders in Bezug auf Totgeburten oder Suizide - manifestieren, und es kann eine Sprache gefunden werden, für die im familiären Rahmen oft nur wenig oder gar kein Raum vorhanden ist. Insofern fördert das Internet eine Demokratisierung der Trauersprache: Hier kann jede Person ihre Trauer und ihren Schmerz auf eigene Art und Weise artikulieren. Außerdem ist in den verschiedenen Trauerforen oftmals zu beobachten, dass Langzeittrauernde sich als Ratgeber:innen für andere (Neu-)Betroffene betätigen und somit eine neue Rolle finden.

Fotografien von Totgeborenen finden sich auf virtuellen Friedhöfen, die entweder ausschließlich für tot geborene Babys eingerichtet wurden ${ }^{24}$ oder die spezielle Abteilungen für Stillborns aufweisen ${ }^{25}$, in verschiedensten (Medien-) Berichten, die online dokumentiert sind ${ }^{26}$, auf zahlreichen privaten FacebookEinträgen, oder auch auf Webseiten, die Eltern eigens für ihre tot geborenen Kinder angelegt haben. ${ }^{27}$

Dabei sind die Fotos wie Grabsteine angeschrieben mit den Namen der toten Babys und mit ihrem in eins fallenden Geburts- und Todesdatum. Meist sieht man nur die Gesichter. Die toten Leiber sind in weiße weiche Kleider eingewickelt, oft tragen sie Wollmützen. Bisweilen sind die Gesichter fleckig, verfärbt, übersät mit Pusteln oder Geschwülsten; die Augen sind stets geschlossen. Selten sieht man einen nackten Oberkörper, und nur in äußersten Fällen gar den nackten Leib mit Totenflecken. Die kleinen Toten liegen in Wiegen oder auf Betten. Bisweilen sind die Liegestätten in digitaler Nachbearbeitung auch als Wolken dargestellt, und manchmal tragen die kleinen Leichen gar Flügelchen.

24 Z. B. https://stillbirthday.com/gestational-age-of-your-baby/ (letzter Zugriff: 23.11.2021).

25 Z. B. https://christianmemorials.com/memorials?filter=category_id\&sort=a-z\&value=2 (letzter Zugriff: 23.11.2021).

26 Z. B. https://www.abc.net.au/news/2016-o9-o5/chloe-the-story-of-a-stillborn-baby/ 7805998?nw=o (letzter Zugriff: 23.11.2021). 
Über neugeborene Tote lässt sich nur schwerlich etwas aussagen. Wenn man sprachgewandt ist, kann man, wie im Falle von Kotzwinkle oder Alice Jolly, mit Worten beschreiben, was man sieht. Aber man kann über die kleinen Toten kaum als Personen sprechen, man kann keine Eigenschaften würdigen, keine Lebensstationen aufzählen, keine Ereignisse zum Besten geben, man kann sie nur zeigen. Ihr wesentlicher Nachruf ist daher das Bild. So beschreiben die Eltern die hergezeigten Fotos ihrer tot geborenen Kinder nur knapp, beispielsweise mittels Bibelverweis, oder indem sie zusätzlich zum angegebenen Geburts- und Todesdatum noch einen Satz über das Krankenhaus hinzufügen, in dem das Kind zur Welt gekommen und gestorben ist, oder es heißt einfach nur: „This is my daughter rosalee.“ In Kommentaren finden sich hauptsächlich Trostworte von anderen, oft handelt es sich um Zuspruch von Personen, die ebenfalls Eltern von tot geborenen Kindern sind (vgl. Abb. 48 bis 53).

Webseiten mit Fotografien toter Babys kamen in der zweiten Hälfte der Nullerjahre im US-amerikanischen und britischen Raum auf, gleichzeitig mit entsprechenden Fotografien auf Social-Media-Kanälen. Zunächst waren auf diesen Fotos einzig tot geborene Babys zu sehen. Im Laufe der Zehnerjahre jedoch wurde zunehmend auch das Abschiednehmen selbst dokumentiert, sodass nun vermehrt auch die Eltern sowie weitere Verwandte mit dem toten Kind gemeinsam ins Bild kamen. Solche Abschiedszeremonien sind ebenfalls auf individuellen Gedenkseiten zu finden sowie auch auf YouTube. ${ }^{28}$

Die öffentliche Archivierung und Ausstellung von Fotos tot geborener Babys ist deshalb besonders bemerkenswert, weil die virtuelle Friedhofskultur des Internets den toten Körper in der Regel leugnet. Gerade hier scheint die Totenfotografie tabu (vgl. Kap. 7, S. 131) - mit Ausnahme eben der Fotos Totgeborener. An dieser Stelle ist die international agierende NGO Now I Lay Me Down to Sleep zu nennen, die zum propagierten Bild des toten Babys wesentlich beiträgt, indem sie weltweit Fotograf:innen vermittelt, welche sich auf das Fotografieren von Stillborn Babies spezialisiert haben. ${ }^{29}$ Damit vollzieht sich eine partielle Rückkehr des Totenfotografie-Genres.

Die Veröffentlichung von Totgeborenen-Fotos im Internet mochte zu Beginn befremdlich erscheinen, doch zweifellos stellt sie mittlerweile einen medialen Trauerort dar, der zunehmend beansprucht wird. Die Eltern zeigen ihre toten

28 www.youtube.com/watch?v=owzvCpB53bE sowie www.youtube.com/watch?v=y4cv2zg UWas. Bisweilen werden auch integral gefilmte Gedenkfeiern gepostet, siehe z. B.: www. youtube.com/watch?v=qvqsldMSQFk (letzter Zugriff jeweils: 23.11.2021).

29 https://www.nowilaymedowntosleep.org/about-us/. Überdies finden sich im Internet Tipps, wie man am besten Erinnerungsbilder von verstorbenen Babys mit Fehlbildungen macht, vgl. dazu etwa https://www.anencephaly.info/d/fotos.php (letzter Zugriff jeweils: 23.11.2021). 


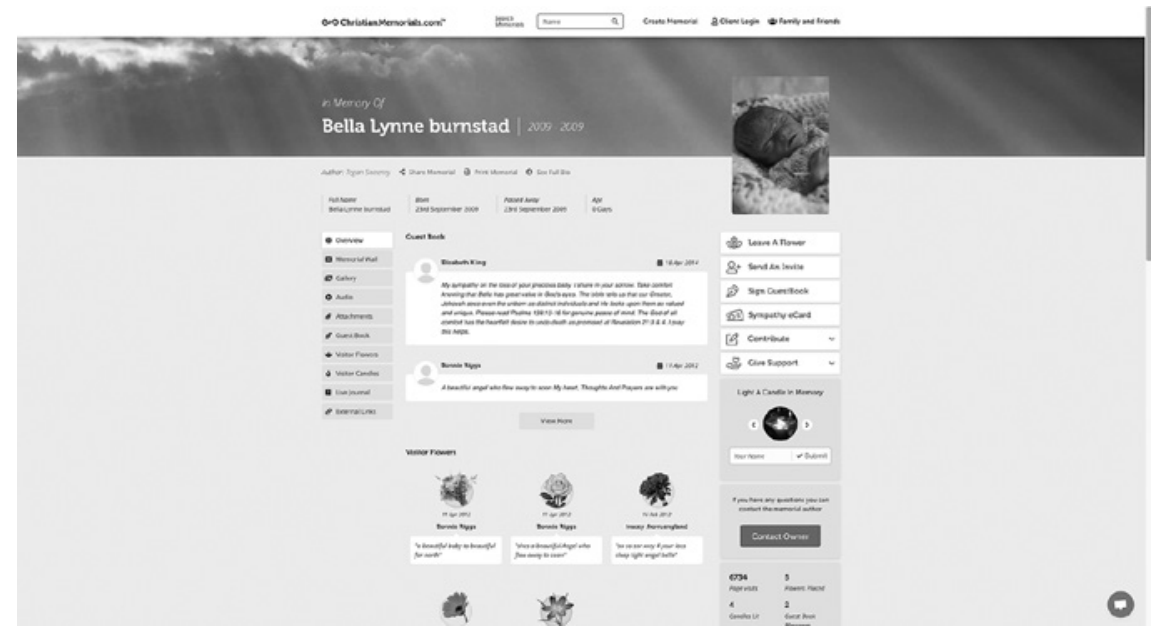

Abb. 48 https://christianmemorials.com/tributes/bella-burnstad (letzter Zugriff: 23.11.2021).

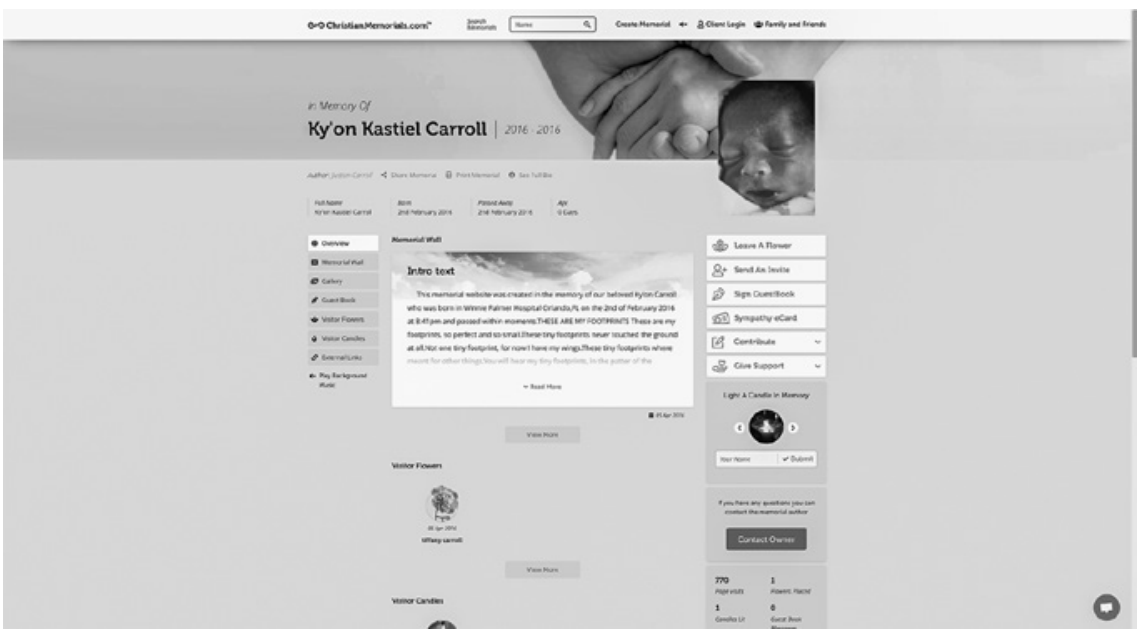

Abb. 49 https://christianmemorials.com/tributes/kyon-carroll (letzter Zugriff: 23.11.2021). 


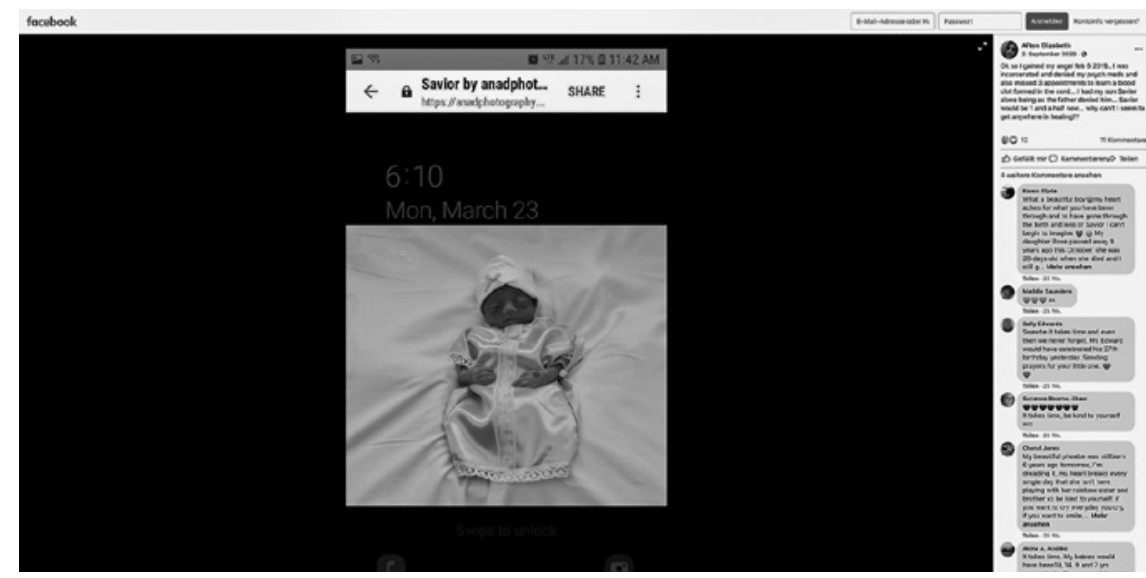

Abb. 5o https://www.facebook.com/photo/?fbid=3856402077707922\&set =g.1422272074671133 (letzter Zugriff: 23.11.2021).

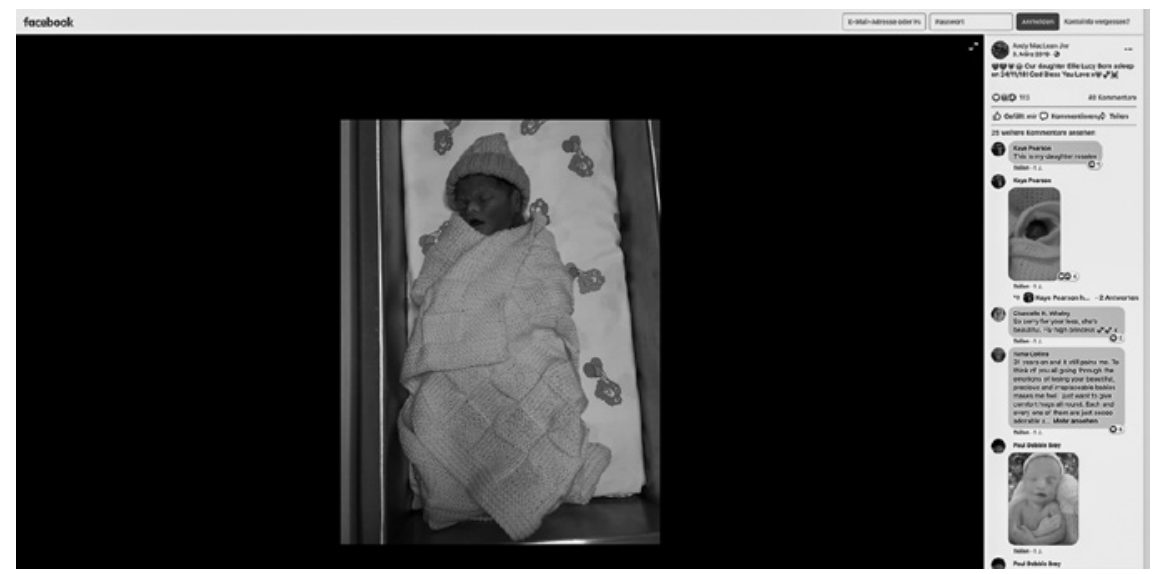

Abb. $5^{1} \quad$ https://www.facebook.com/photo?fbid=330343697590997\&set=g.142227207467113 (letzter Zugriff: 23.11.2021). 


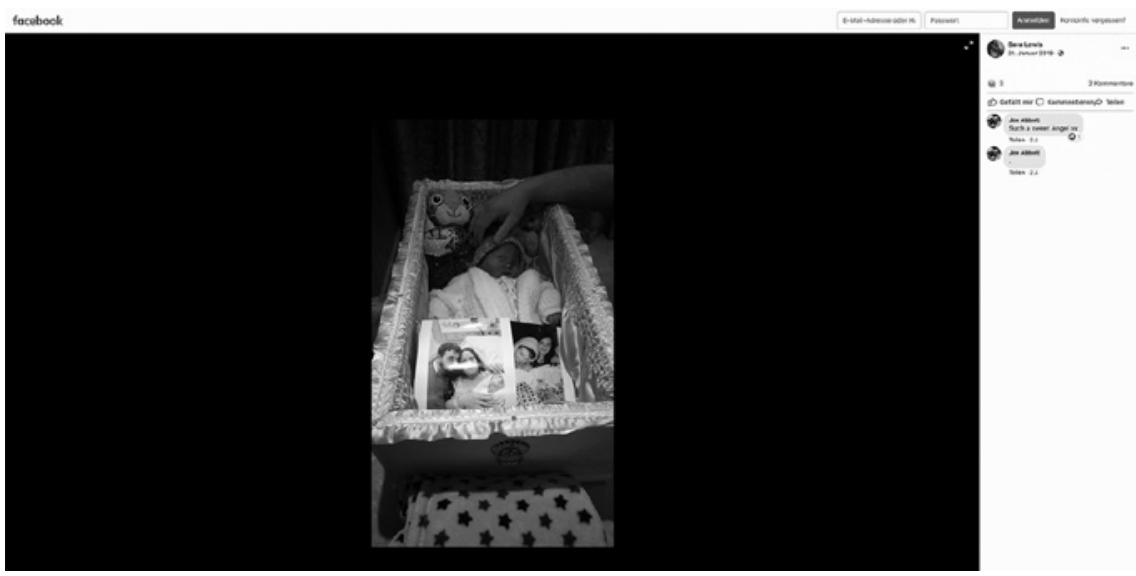

Abb. $5^{2}$ https://www.facebook.com/photo?fbid=10217531175448574\&set =g.1422272074671133 (letzter Zugriff: 23.11.2021).

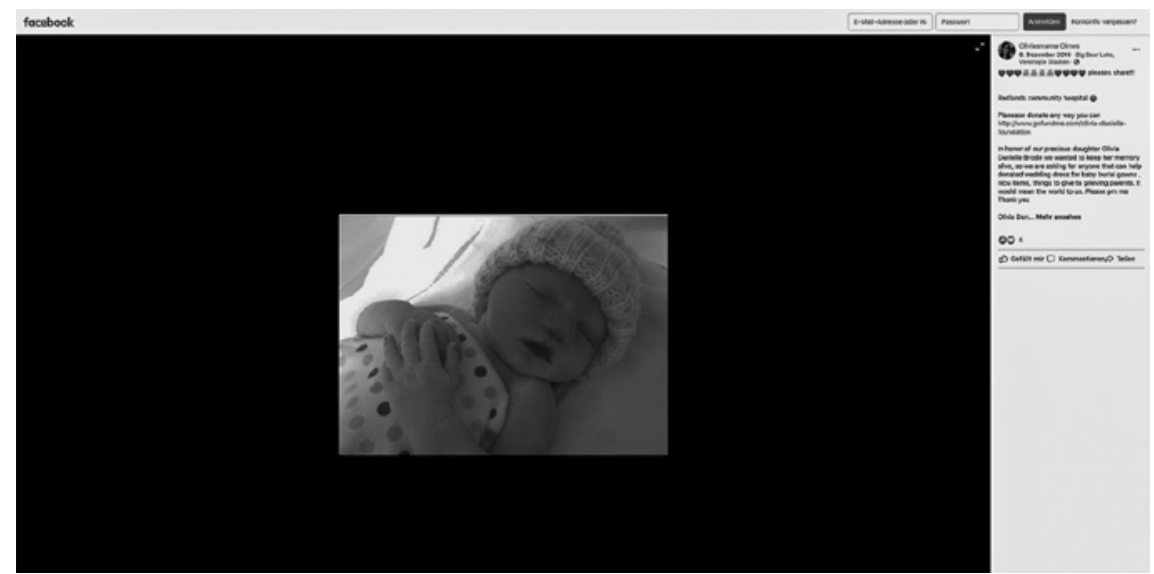

Abb. 53 https://www.facebook.com/photo/?fbid=10208141796130461\&set=g.26307302976 (letzter Zugriff: 23.11.2021). 
Kinder mit der öffentlich bezeugten Gebärde des Wahrhabenwollens; es ist eine Gebärde, die das Medium hervorbringt. Hierbei finden sich auch Eintragungen von Babynamen, die vor zwanzig, dreißig, ja vierzig Jahren tot zur Welt gekommen sind. Stellt man das Bild eines tot geborenen Babys der Öffentlichkeit zur Verfügung, so begünstigt dies auch nachträglich den Prozess, ein Selbstverständnis als Mutter oder Vater eines toten Kindes anzunehmen ohne sich dessen, im Schutze all der anderen Eltern auf der jeweiligen Webseite, zu schämen. Denn wenn ein Kind stirbt, sei es bei der Geburt oder später, so haben die Eltern, insbesondere die Mütter, oftmals mit Scham und Schuldgefühlen zu kämpfen, wovon insbesondere die autobiografischen Darstellungen zeugen. Für die Trauerbewältigung stellt das veröffentlichte Foto zweifellos eine zentrale Erinnerungshilfe dar. 